\title{
A!
}

This is an electronic reprint of the original article.

This reprint may differ from the original in pagination and typographic detail.

Nefedov, Igor S.; Melnikov, Leonid A.

\section{Super-Planckian far-zone thermal emission from asymmetric hyperbolic metamaterials}

Published in:

Applied Physics Letters

DOI:

$10.1063 / 1.4899126$

Published: 01/01/2014

Document Version

Publisher's PDF, also known as Version of record

Please cite the original version:

Nefedov, I. S., \& Melnikov, L. A. (2014). Super-Planckian far-zone thermal emission from asymmetric hyperbolic metamaterials. Applied Physics Letters, 105(16). https://doi.org/10.1063/1.4899126

This material is protected by copyright and other intellectual property rights, and duplication or sale of all or part of any of the repository collections is not permitted, except that material may be duplicated by you for your research use or educational purposes in electronic or print form. You must obtain permission for any other use. Electronic or print copies may not be offered, whether for sale or otherwise to anyone who is not an authorised user. 


\section{Super-Planckian far-zone thermal emission from asymmetric hyperbolic metamaterials}

Igor S. Nefedov, and Leonid A. Melnikov

Citation: Appl. Phys. Lett. 105, 161902 (2014); doi: 10.1063/1.4899126

View online: https://doi.org/10.1063/1.4899126

View Table of Contents: http://aip.scitation.org/toc/apl/105/16

Published by the American Institute of Physics

\section{Articles you may be interested in}

Broadband super-Planckian thermal emission from hyperbolic metamaterials

Applied Physics Letters 101, 131106 (2012); 10.1063/1.4754616

Super-Planckian near-field thermal emission with phonon-polaritonic hyperbolic metamaterials Applied Physics Letters 102, 131106 (2013); 10.1063/1.4800233

Near-field radiative thermal transport: From theory to experiment AlP Advances 5, 053503 (2015); 10.1063/1.4919048

Three-dimensional photonic-crystal emitter for thermal photovoltaic power generation Applied Physics Letters 83, 380 (2003); 10.1063/1.1592614

Near-field thermal radiation between hyperbolic metamaterials: Graphite and carbon nanotubes Applied Physics Letters 103, 213102 (2013); 10.1063/1.4832057

Graphene-assisted near-field radiative heat transfer between corrugated polar materials Applied Physics Letters 104, 251911 (2014); 10.1063/1.4885396

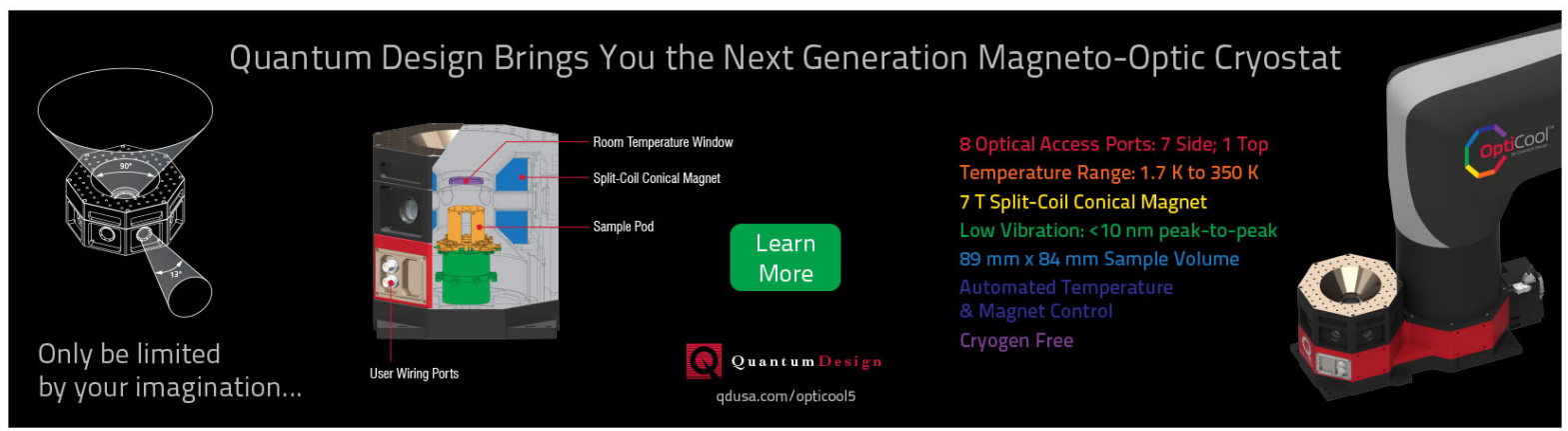




\title{
Super-Planckian far-zone thermal emission from asymmetric hyperbolic metamaterials
}

\author{
Igor S. Nefedov ${ }^{1}$ and Leonid A. Melnikov ${ }^{2}$ \\ ${ }^{1}$ School of Electrical Engineering, Aalto University, P.O. Box 13000, 00076 Aalto, Finland \\ ${ }^{2}$ Yuri Gagarin State Technical University of Saratov, 77 Politekhnicheskaya, 410054 Saratov, Russia
}

(Received 23 June 2014; accepted 11 October 2014; published online 20 October 2014)

\begin{abstract}
We demonstrate the production of strong directive thermal emissions in the far-field zone of asymmetric hyperbolic metamaterials (AHMs), exceeding that predicted by Planck's limit. Asymmetry is inherent to the uniaxial medium, where the optical axis is tilted with respect to medium interfaces. The use of AHMs is shown to enhance the free-space coupling efficiency of thermally radiated waves, resulting in Super-Planckian far-field thermal emission in certain directions. This effect is impossible in usual hyperbolic materials because emission of high density of states (DOS) photons into vacuum with smaller DOS is preserved by the total internal reflection. Different plasmonic metamaterials are proposed for realizing AHM media; the thermal emission from a AHM, based on a grapheme multilayer structure, is presented, as an example. (C) 2014 AIP Publishing LLC.

[http://dx.doi.org/10.1063/1.4899126]
\end{abstract}

Thermal emitters, including the black body, are commonly considered as incoherent light sources. However, soon after 2000 the selective and partially coherent thermal emission was demonstrated in the far zone from threedimensional photonic crystals ${ }^{1}$ and grating. ${ }^{2}$ Since then many structures were proposed to create directive thermal emitters. $^{3-10}$ These relatively simple sources of coherent emission can be applied in photonics as alternatives to such mid-infrared sources as light-emitting diodes and quantum cascade lasers.

After the works of Kirchhoff (1859-1862) and Planck (1900) the black body (BB) was considered as a perfect thermal emitter with emissivity depending only on the temperature at a certain frequency, and the limit which cannot be exceeded. Rytov ${ }^{11}$ and Polder and Van Hove ${ }^{12}$ have shown that the black-body limit for the thermal radiation heat transfer between two hot bodies, separated by nanometer gaps, can be significantly exceeded due to photon tunneling. This effect was applied for near-field thermophotovoltaic systems. ${ }^{13}$ Pendry has shown that the dramatic enhancement holds due to excitation of coupled surface-plasmon polaritons at interfaces of two media. ${ }^{14}$ Nefedov and Simovski ${ }^{15}$ proposed a way to extend the given effect from nanoscale to micrometer distances using the media, possessing hyperbolic dispersion, i.e., the hyperbolic media (HM). Exploitation of HM seems to be promising for enhancement of the near-field thermal radiative heat transfer. ${ }^{16-20}$ However, according to existing concepts, the black body restriction cannot be overcome in the far zone. In 2003, Lin, Moreno, and Fleming reported that the $\mathrm{BB}$ limit was exceeded in their $3 \mathrm{D}$ photonic crystal emitter. ${ }^{21}$ Then, Trupke et $a l .^{22}$ criticized the work asserting the violation of the thermodynamics law: "There is no doubt that the density of states for certain photons and with it the photon density can be very large in a photonic crystal. The problem is to get these photons out. Emission of all photons into the smaller number of states in a vacuum would require an increase of the occupation probability, equivalent to reduced entropy. Nature avoids this violation of the second law by totally reflecting those photons, which have no corresponding states in the outside medium." "What we claim here is that the photon flux thermally emitted by a body in a certain direction and in a given photon energy interval, whatever the geometry of that body, cannot exceed the photon flux thermally emitted by a black body of the same temperature, in much the same way as the absorbency of a black body, cannot further be improved to exceed unity."22 This point of view has achieved widespread acceptance.

Impossibility of the Super-Planckian thermal emission from a flat surface has a theoretical background which, in our case as is shown below, is not applicable. In the papers, ${ }^{23-27}$ the so-called general approach has been developed for the radiative heat transfer, thermal emission, and the van der Waals friction in the arbitrary-anisotropic media. This approach is based on the transformation of an integral over the volume of the body to an integral over the surface of the body. Using the connection between the vacuum and material parts of Green's function via the Fresnel coefficients, the thermal emission is expressed through the vacuum part of Green's function which gives expression for the thermal emission in the form of the classical Kirchhoff law. However, in Ref. 23 despite the initial statement of the problem the authors starting from the Maxwell equations for arbitrary-anisotropic media at a certain step suppose the electric field correlations $\left\langle E_{x} E_{z}^{*}\right\rangle,\left\langle E_{y} E_{z}^{*}\right\rangle$ (see Eq. (B19)) are taken to be zero which makes the subsequent derivation valid for such anisotropy where both permittivity tensor components $\epsilon_{x z}, \epsilon_{y z}$ are equal to zero, i.e., the anisotropy axis is either orthogonal or parallel to interface. Thus, this formalism is not valid for the media with nonzero $\epsilon_{x z}$ as is in our case. In the following papers, ${ }^{24-27}$ the authors directly refer to Ref. 23 or use similar assumptions.

We do not discuss whether the Super-Planckian emission was really observed in Ref. 21 . We refer a reader to the old Rytov's paper ${ }^{28}$ where he provided theoretical evidence that a sphere with the diameter, compared to the wavelength, can radiate several times more than BB. Recently, 
the Super-Planckian far-zone emission from long cylindrical objects was theoretically predicted in Ref. 29 and was experimentally demonstrated from the surface, covered with the transparent (non-emitting) lens transforming part of the spatial spectrum of evanescent waves into propagating in the free space. ${ }^{30}$ Note that the reported way of enhancing the far-field thermal emission contradicts the above Trupke argumentation since some photons, "which have no corresponding states in the outside medium" can be emitted into the free space via the lens. In this paper, we will show how very high density of states (DOS) of photons, existing in HM, can get out without the total internal reflection exploiting AHMs which are metamaterials having anisotropy axes tilted to interfaces (see the schematic view in Fig. 1(a)).

Let us suppose that the homogeneous isotropic medium (IM) and HM are separated by an interface plane. Thus, depending on the dielectric constants and interface orientation according to the optical axis of HM, the waves can propagate in both media. To calculate the characteristics of thermal radiation, it is necessary to find the eigenmodes of the whole structure, together with the dispersion relation for the frequency and density of modes. ${ }^{31}$ In the box $l_{x} \times l_{y} \times l_{z}$ with ideally reflecting walls in which IM occupies the volume $-l_{z} / 2<z<0$, while HM fill the rest of the cuboid $0<z<l_{z} / 2$, the field can be presented as the plane waves with the wave vector $\vec{k}=\left(k_{x}, k_{y}, k_{z}\right)$ in IM-part and wave vector $\vec{k}_{\mathrm{HM}}$ $=\left(k_{x}, k_{y}, k_{z}^{\mathrm{HM}}\right)$ in HM-part. The HM optical axis is tilted according to the interface plane by the angle $\phi$. Each mode "dwells" in both IM and HM, and the quanta of field are spread among the media depending on the interface orientation and $\vec{k}$, which parametrises the eigenmodes. Providing the thermally excited field oscillators, the equilibrium state is achieved having Planck's average energy of the oscillator, though the spatial distribution and energy can be other than in the BB radiation. Dispersion equations for $\mathrm{IM}$ and $\mathrm{HM}$ are correspondingly ${ }^{32} \omega_{\mathrm{IM}}(k)=c k \epsilon$, $\omega_{\mathrm{HM}}(k)=c k \sqrt{\frac{1}{2}\left[\epsilon_{\perp}^{-1}+\epsilon_{z}^{-1}+\left(\epsilon_{\perp}^{-1}-\epsilon_{z}^{-1}\right) \cos 2 \Phi\right]} . \Phi$ is the azimuthal angle between $\vec{k}$ and optical axis. Here, both $\epsilon_{\perp, z}$ are supposed to be frequency independent (dispersion can be

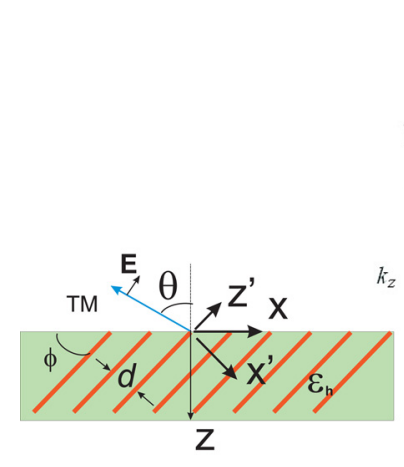

(a)

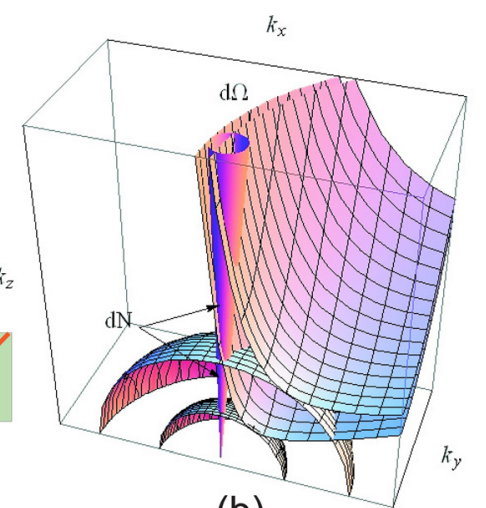

(b)
FIG. 1. (a) A schematic view of the hyperbolic metamaterial with a tilted optical axis. Here, $d$ is the lattice period of a metamaterial. (b) The elliptic and hyperbolic dispersion surfaces in $3 \mathrm{D}$ space, corresponding to the same frequency. incorporated easily, and further we will use frequency dependent components for $\hat{\epsilon})$. We can write: $\omega_{\mathrm{IM}}^{2}=\omega_{\mathrm{HM}}^{2}$ $\rightarrow k_{z, 1,2}^{\mathrm{HM}}=\sec 2 \phi\left( \pm \sqrt{k_{x}^{2}+\left(k_{x}^{2}+2 k_{y}^{2}+k_{z}^{2}\right) \cos 2 \phi}-k_{x} \sin 2 \phi\right)$ for $\epsilon_{\perp}=1, \epsilon_{z}=-1$. The accumulated phase during the propagation from $-l_{z} / 2$ to $l_{z} / 2$ is to be equal to $2 \pi n_{z}$ for downward and upward waves

$$
\begin{gathered}
\left(l_{z} / 2\right)\left(k_{z}+k_{z, 1}^{\mathrm{HM}}\left(k_{x}, k_{y}, k_{z}\right)\right)=2 \pi n_{z}, \\
2 \pi n_{z}=\left(l_{z} / 2\right)\left(k_{z}+k_{z, 2}^{\mathrm{HM}}\left(k_{x}, k_{y}, k_{z}\right)\right) .
\end{gathered}
$$

When $k_{z} \gg k_{x}, k_{y}$ (for $\epsilon_{\perp}=1, \epsilon_{z}=-1$ ) the solution is given by

$$
(2 \pi)^{-1} k_{z} \approx 2 n_{z}(\sqrt{\cos 2 \phi}-\cos 2 \phi) /(1-\cos 2 \phi),
$$

which can be used to calculate of DOS: $d N=d n_{x} d n_{y} d n_{z}$ $=(2 \pi)^{-3} d k_{x} d k_{y} d k_{z}(1-\cos 2 \phi) /(\sqrt{\cos 2 \phi}-\cos 2 \phi), l_{x} l_{y} l_{z}=1$. It means that DOS goes to infinity where $\phi \rightarrow \pi / 4$. In this particular case, the physical reason for the divergence of DOS is $k_{z} \rightarrow \infty$ if $k_{x}=k / \sqrt{2}$ and the parameter of losses tends to zero, see Refs. 33-36. Thus, combining IM and AHM we can obtain the medium with a higher DOS corresponding to the propagating modes than in the vacuum part only. Field energy distribution among IM and AHM parts depends on the reflection at the interface plane.

In AHMs, the reflection can be small enough to manifest the DOS increase. Such metamaterials are characterized by the asymmetry in the properties of wave, propagating upward and downward with respect to the interfaces under a fixed transverse component of the wave vector, and exhibit unique absorbing properties (see Refs. 33-36). Fig. 1(b) shows the spherical and hyperbolic isofrequency surfaces, calculated for the same frequency. The number of states $d N$ is proportional to the volume, enclosed in the solid angle $d \Omega$ between two isofrequencies. Projection of this volume onto the $\left(k_{x} k_{y}\right)$-plane belongs to the area of the propagating waves. It means that the waves with very large modules of the wave vector can leave AHM without the total internal reflection, and high DOS photons in AHM are coupled with photons in the free space, while the usual reflection is quite moderate and does not suppress the far-field SP emission.

We assume that the medium is semi-infinite in the $z$ direction (see Fig. 1(a)). The permittivity tensor in the interface coordinate system $\mathrm{XOZ}$ can be expressed via the rotation transformation, see Ref. 33. Since conventional formulas expressing thermal emission (see, for example, Refs. 23, 37, and 38) are not applicable for the media with $\epsilon_{x z} \neq 0 ; \epsilon_{y z} \neq 0$, we start as in Ref. 12 from the nonhomogeneous Maxwell equations with the random electric current density sources $\mathbf{j}$ and apply the fluctuation-dissipation theorem ${ }^{39}$ for the ensemble-averaged bulk current density given by

$$
\begin{aligned}
& \left\langle j_{m}(\mathbf{r}, \omega) j_{n}^{*}\left(\mathbf{r}^{\prime}, \omega^{\prime}\right)\right\rangle \\
& \quad=\frac{4}{\pi} \omega \epsilon_{0} \epsilon_{m n}^{\prime \prime}(\omega) \delta\left(\mathbf{r}-\mathbf{r}^{\prime}\right) \delta\left(\omega-\omega^{\prime}\right) \Theta(\omega, T),
\end{aligned}
$$

where $\Theta(\omega, T)$ is Planck's oscillator energy. In Eq. (3), $\epsilon_{m n}^{\prime \prime} \equiv \operatorname{Im}\left(\epsilon_{m n}\right), j_{m}$ and $j_{n}(m, n=1,2$, or 3$)$ are $x, y$, or $z$ component of $\mathbf{j}, \delta(x)$ is the Dirac delta function, $\epsilon_{0}$ is the permittivity of vacuum. The omitted time dependence is $e^{-i \omega t}$, an 
elementary bulk current source can be given as $\mathbf{j}(x, z)=\mathbf{j}_{0}\left(x^{\prime}, z^{\prime}\right) \delta(\bar{x}) \delta(\bar{z})$, where $\bar{x}=x-x^{\prime}, \bar{z}=z-z^{\prime}$.

For any uniaxial crystal, Maxwell equations are split into two sub-systems describing the ordinary and extraordinary waves. The hyperbolic electric medium supports the propagation of TM (p-polarized) waves only for a whole spectrum of transversal wave vectors, which are extraordinary. We restrict our consideration by the TM-waves propagating in the $(x z)$-plane $(\partial / \partial y=0)$. In this case, the electric field vector lies in plane containing the anisotropy axis and $z$ axis. This plane is orthogonal to the interface, and both reflected and transmitted waves keep the TM-polarization.

Let us use the spectral representation for the current and nonzero field components for the TM-polarized wave in the bulk AHM

$$
\left[\begin{array}{c}
\mathbf{j}(x, z) \\
E_{x}(x, z) \\
H_{y}(x, z)
\end{array}\right]=\iint_{-\infty}^{\infty}\left[\begin{array}{c}
\mathbf{j}_{0}\left(x^{\prime}, z^{\prime}\right) \\
e_{x}(\alpha, \beta) \\
h_{y}(\alpha, \beta)
\end{array}\right] \frac{e^{i[\alpha \bar{x}+\beta \bar{z}]}}{(2 \pi)^{2}} d \alpha d \beta .
$$

Here, we included the exponent $e^{-i\left(\alpha x^{\prime}+\beta z^{\prime}\right)}$ into the unknown Fourier transforms $e_{x}(\alpha, \beta), h_{y}(\alpha, \beta)$ taking into account the Fourier transform of the delta-functions. In the Fourier space fields, created at the point $(x, z)$ by the source located at $\left(x^{\prime}\right.$, $z^{\prime}$ ), are given by

$$
\begin{aligned}
& e_{x}(\alpha, \beta)=\frac{i}{\Delta}\left[-j_{0 x}\left(x^{\prime}, z^{\prime}\right) A_{22}+j_{0 z}\left(x^{\prime}, z^{\prime}\right) A_{12}\right], \\
& h_{y}(\alpha, \beta)=\frac{i}{\Delta}\left[-j_{0 x}\left(x^{\prime}, z^{\prime}\right) A_{21}+j_{0 z}\left(x^{\prime}, z^{\prime}\right) A_{11}\right],
\end{aligned}
$$

where $\rho=120 \pi$ is the vacuum wave impedance,

$$
\begin{gathered}
\Delta=A_{11} A_{22}-A_{12} A_{21}, \\
A_{11}=\frac{k_{0}}{\eta}\left(\epsilon_{x x}+\frac{\beta}{\alpha} \epsilon_{x z}\right) A_{12}=-\left(\beta+\frac{k_{0}^{2}}{\alpha} \epsilon_{x z}\right) \\
A_{21}=\frac{k_{0}}{\eta}\left(\epsilon_{x z}+\frac{\beta}{\alpha} \epsilon_{z z}\right) A_{22}=\alpha-\frac{k_{0}^{2}}{\alpha} \epsilon_{z z} .
\end{gathered}
$$

Note that the condition $\Delta(\alpha)=0$ is actually the dispersion equation for the TM waves in the unbounded medium.

Let us substitute (5) in Eq. (4) and evaluate the integral over $\beta$ using the residuum method. The poles of integrands (zeros of the equation $\Delta\left(\beta_{m}\right)=0$ ) are ar $^{32,33}$

$$
\beta_{1,2}=k_{z}^{(1,2)}=\frac{\alpha \epsilon_{x z} \pm \sqrt{\left(\epsilon_{x z}^{2}-\epsilon_{x x} \epsilon_{z z}\right)\left(\alpha^{2}-k_{0}^{2} \epsilon_{z z}\right)}}{\epsilon_{z z}} .
$$

(A number of certain interesting features of these solutions for hyperbolic media are described in Refs. 32-35.) We close the integration contour in the lower half-space of the complex plane, choosing the zero $\Delta(\alpha)$ where $\Im\left(k_{z}^{(i)}\right)<0$ corresponds to the wave attenuating in the positive direction of the $z$-axis at $z<0$.

Application of the residuum method results in the following expressions for the field components at the point $(x, z)$, excited by the point-like current located at the point $\left(x^{\prime}, z^{\prime}\right)$ :

$$
\left[\begin{array}{c}
E_{x} \\
H_{y}
\end{array}\right]=\int_{-\infty}^{\infty}\left[\begin{array}{c}
\tilde{e}_{x}(\alpha, z) \\
\tilde{h}_{y}(\alpha, z)
\end{array}\right] e^{\alpha(\bar{x})} \frac{e^{-i k_{z}^{(m)}|\bar{z}|}}{2 \pi} d \alpha
$$

$$
\begin{aligned}
& \tilde{e}_{x}(\alpha, z)=\frac{1}{\Delta^{\prime}}\left[-j_{0 x}\left(x^{\prime}, z^{\prime}\right) \tilde{A}_{22}(\alpha)+j_{0 z}\left(x^{\prime}, z^{\prime}\right) \tilde{A}_{12}(\alpha)\right] \\
& \tilde{h}_{y}(\alpha, z)=\frac{1}{\Delta^{\prime}}\left[-j_{0 x}\left(x^{\prime}, z^{\prime}\right) \tilde{A}_{21}(\alpha)+j_{0 z}\left(x^{\prime}, z^{\prime}\right) \tilde{A}_{11}(\alpha)\right]
\end{aligned}
$$

where $\Im\left(k_{z}^{(m)}\right) \leq 0$ and the expressions for $\tilde{A}_{i j}$ are obtained from (7) substituting $k_{z}(\alpha)$ instead of $\beta$. Here, the superscript $m$ omitted in the notation for $k_{z}$ and

$$
\Delta^{\prime}\left(k_{z}^{(m)}\right)=\frac{d}{d \beta} \Delta(\beta)_{\mid \beta=k_{z}^{(i)}}=\frac{2 k_{0}}{\eta}\left(\epsilon_{x z}+\frac{k_{z}}{\alpha} \epsilon_{z z}\right) .
$$

Therefore, the formulas (9) and (10) give the expressions for the corresponding components of dyadic Green's functions.

Then from the field components we compose the quadratic form $E_{x}\left(x, z \mid x^{\prime}, z^{\prime}\right) H_{y}^{*}\left(x, z \mid x^{\prime \prime}, z^{\prime \prime}\right)$, corresponding to the Poynting vector, and integrate it over all the distributed sources, located in the points $\left(x^{\prime}, z^{\prime}\right)$ and $\left(x^{\prime \prime}, z^{\prime \prime}\right)$. Evaluating the integration and using (3), we obtain the expression for the ensemble-averaged Poynting vector, incident onto the interface $z=0$ :

$$
\left\langle S_{z}(x, \omega)\right\rangle=\int_{-\infty}^{\infty}\left\langle S_{z}\left(k_{x}, \omega\right)\right\rangle d k_{x},
$$

where

$$
\begin{aligned}
\left\langle S_{z}\left(k_{x}, \omega\right)\right\rangle= & \frac{k_{0}}{\pi \eta} \frac{\Im\left(k_{z}\right)}{\left|\Delta^{\prime}\right|^{2}}\left[\epsilon_{x x}^{\prime \prime} \tilde{A}_{22} \tilde{A}_{21}^{*}+\epsilon_{z z}^{\prime \prime} \tilde{A}_{12} \tilde{A}_{11}^{*}\right. \\
& \left.-\epsilon_{x z}^{\prime \prime}\left(\tilde{A}_{22} \tilde{A}_{11}^{*}+\tilde{A}_{12} \tilde{A}_{21}^{*}\right)\right] .
\end{aligned}
$$

Thus, we have obtained the Poynting vector, coming to the interface and created by the fluctuating currents distributed in the halve-space $z>0$. The boundary conditions are taken into account via the transmission coefficient ${ }^{15}$ $t_{s}=|t|^{2} Z_{t}^{*} / Z_{0}^{*}$, where $Z_{0}$ and $Z_{t}$ are the transverse wave impedances for the vacuum and asymmetric hyperbolic medium, respectively, and $t$ is the transmission coefficient, defined for the electric field vector. These expressions are given in Refs. 33 and 35 . Note that $Z_{t}^{(1,2)}= \pm Z_{t}$, corresponding to $k_{z}^{(1,2)}$, differ only in sign. Thus, the spectral density of the thermal emission flux radiated at the angle $\theta$ into the plane angle $d \theta$ can be given as

$$
q(\omega, \theta)=\left\langle S_{z}\left(k_{x}, \omega\right)\right\rangle t_{s} \cos \theta+\text { c.c. }
$$

If we assume that $\epsilon_{x z}=0$ (and, correspondently, $\left\langle E_{x} E_{z}^{*}\right\rangle=0$ ), Eqs. (13) and (14) are reduced to the form of the conventional Kirchhoff law.

As an example of AHM, we consider a graphene multilayer (GM) which is the structure consisting of periodically arranged graphene sheets embedded into a host matrix with the relative permittivity $\epsilon_{h}$ and tilted with respect to the interface. For the relative transverse tensor component $\epsilon_{t}$ we used the homogenization model $\epsilon_{\perp}=\epsilon_{h}+i \frac{\sigma}{d \omega \epsilon_{0}}$, where $\sigma$ is the graphene conductivity calculated by the Kubo formula ${ }^{40}$ and $d$ is the multilayer lattice constant. The longitudinal tensor component $\epsilon_{z}$ is equal to $\epsilon_{h}$. Under certain conditions, the graphene multilayers exhibit properties specific for the hyperbolic media. ${ }^{36,41}$ 


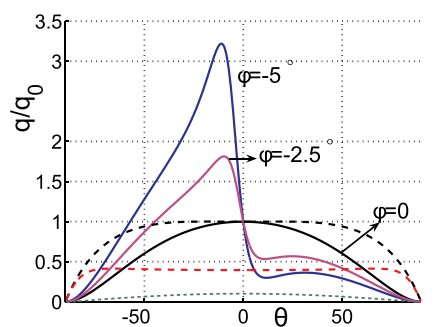

(a)

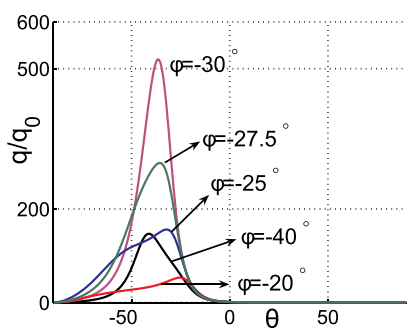

(b)
FIG. 2. Normalized emissivity. The dashed curves show the transmission modulus for the Poynting vector. The dotted green curve shows normalized emissivity of the TE-polarized wave.

Figs. 2(a) and 2(b) illustrate the spectral density of the thermal emission flux from the graphene multilayers with different tilt angles $\phi$ into the plane angle. Thermal emission is normalized to the same characteristic of the black body $q_{\mathrm{BB}}(\omega, \theta)$. The parameters of $\mathrm{GM}$ are the following: $d=10 \mathrm{~nm} ; \epsilon_{h}=1.2+i 0.01$; the relaxation time of the carriers is $10^{-13} \mathrm{~s}$, the chemical potential of graphene is $\mu_{c}=0.15 \mathrm{eV}$, which can be provided by applying the voltage, and the wavelength $\lambda=8 \mu \mathrm{m}$. Under such parameters $\epsilon_{\perp}=-1.3617+i 0.7622$. Fig. 2(a) shows the emission, produced by GM with small tilt angles and vertically standing sheets (red curve), thus the permittivity tensor is diagonal or has small non-diagonal components. The transmission coefficient for the Poynting vector is shown also.

For the tilt angles $\phi=0,2.5^{\circ}$ and $5^{\circ}$, the transmission coefficients are very close and are shown by the black dashed curve. For $\phi=90^{\circ}$, the transmission is significantly lower (see the red dashed curve). Super-Planckian radiation appears at the small tilt angle $2.5^{\circ}$ and its maximum increases with $\phi$. The thermal emission from GM at larger $\phi$ is significantly higher, see Fig. 2(b). There is an optimum which depends on the parameters of GM. The directivity of thermal emission in the polar coordinates is shown in Fig. 3(a).

Mathematical explanation of the SP radiation follows from the formula (13). The angular maxima of the emission correspond to the minima $\left|\Delta^{\prime}(\theta)\right|$, which is going to zero when the material losses are decreased. The given situation is observed in any media. For the isotropic medium with the scalar permittivity $\epsilon$, the field components excited by a point-like source in the medium, are inversely proportional to $k_{z}^{\text {is }}=\sqrt{k_{0}^{2} \epsilon-k_{x}^{2}} \cdot{ }^{32}$ Thus, the $k_{x}$-Fourier component goes to

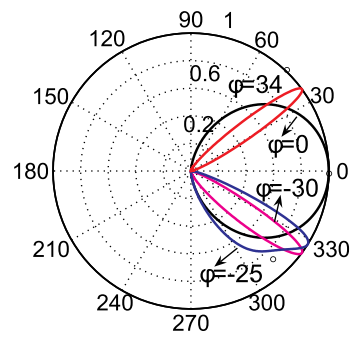

(a)

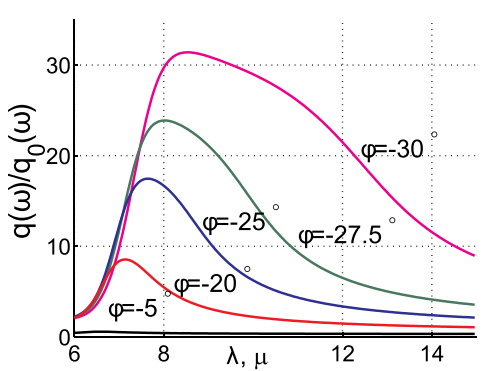

(b)
FIG. 3. (a) Radiation pattern in polar coordinates. (b) Wavelength dependence of the normalized emissivity. infinity if $\operatorname{Im}(\epsilon) \rightarrow 0$ and $\left|k_{x}\right| \rightarrow k_{0} \sqrt{\epsilon}$. However, waves belonging to the spatial spectrum area $k_{x} \approx k_{0} \sqrt{\epsilon}$, always undergo total internal reflection at an interface with the vacuum. It follows from expression for the reflection coefficient which reads for the TM-waves as ${ }^{12} R=\left|\epsilon \gamma-k_{z}^{\text {is }}\right|^{2} /\left|\epsilon \gamma+k_{z}^{\text {is }}\right|^{2}$, where $\gamma=\sqrt{k_{0}^{2}-k_{x}^{2}}$. Similar situation takes place in the anisotropic media if the anisotropy axis is either parallel or orthogonal to the media interfaces. On the contrary, in AHM the minimum $\left|\Delta^{\prime}\left(k_{x}\right)\right|$ falls to the propagating area of the vacuum waves, $\left|k_{x}\right|<k_{0}$ and $\left\langle S_{z}\left(k_{x}, \omega\right)\right\rangle \rightarrow \infty$ at the minimum of $\left|\Delta^{\prime}\left(k_{x}\right)\right|$ if $\operatorname{Im}\left(\epsilon_{z}\right) \rightarrow 0, \operatorname{Im}\left(\epsilon_{\perp}\right) \rightarrow 0$, while the reflection can be minimized. ${ }^{35}$

Fig. 3(b) shows the thermal emission for the TMpolarized waves into the plane angle in the $(x z)$-plane integrated over the emission angle $\theta,-90^{\circ} \leq \theta \leq 90^{\circ}$. Thus, we obtained the emission into a full plane angle, exceeding the black body spectrum by 30 .

Finally, we discuss violation of Kirchhoff's law. It is well-known that a plasmonic resonant particle can absorb more than the light incident on it. ${ }^{42,43}$ Therefore, strong absorption is associated with excitation of surface plasmons or surface phonons ${ }^{42}$ and is inversely proportional to $\epsilon^{\prime \prime}$ at a resonant frequency. (This occurs in the case of thermal emission from AHM.) We can note that the reverse side of "absorption more than $100 \%$ " is the Super-Planckian thermal emission produced by the same particle in the far-field zone. In this case, the conventional Kirchhoff law was generalized introducing the effective absorption cross-section. ${ }^{11} \mathrm{~A}$ similar effect is not present in the conventional bulk media due to impossibility to excite slow surface plasmonic polaritons by the fast waves incoming from the free space. However, bulk plasmons can be excited in asymmetric hyperbolic media by external radiation. ${ }^{33-36}$ The Super-Planckian radiation effect from AHM does not violate the thermodynamics laws, together with SP described in Refs. 28 and 30 radiation. Enhanced emission requires increased thermal flow supply to a radiating body in order to maintain the constant temperature.

Concluding the results of our research are based on the analytical approach which exploits a relatively simple scheme described by D. Polder and M. Van Hove and is easy to reproduce.

${ }^{1}$ S. Y. Lin, J. G. Fleming, E. Chow, J. Bur, K. K. Choi, and A. Goldberg, Phys. Rev. B 62, R2243 (2000).

${ }^{2}$ J. J. Greffet, R. Garminati, K. Joulain, J.-P. Mulet, S. Maingui, and Y. Chen, Nature 416, 61 (2002).

${ }^{3}$ A. Narayanaswamy and G. Chen, Phys. Rev. B 70, 125101 (2004).

${ }^{4}$ K. Joulain, J.-P. Mulet, F. Marquier, R. Carminati, and J.-J. Greffet, Surf. Sci. Rep. 57, 59 (2005).

${ }^{5}$ H. Xin, Z. Wu, A. Young, and R. Ziolkowski, IEEE Trans. Antennas Propag. 56, 2970 (2008).

${ }^{6}$ C. J. Schuler, C. Wolff, K. Busch, and M. Florescu, Appl. Phys. Lett. 95, 241103 (2009).

${ }^{7}$ M. Florescu and K. Busch, J. Opt. A: Pure Appl. Opt. 11, 114005 (2009).

${ }^{8}$ J. Drevillon, K. Joulian, P. Ben-Abdallah, and E. Nefzaoui, J. Appl. Phys. 109, 034315 (2011).

${ }^{9}$ G. D’Aguanno, N. Mattiucci, A. Alu, C. Argyropoulos, J. V. Foreman, and M. J. Bloemer, Opt. Express 20, 9784 (2012).

${ }^{10}$ N. Mattiucci, G. D'Aguanno, A. Alu, C. Argyropoulos, J. V. Foreman, and M. J. Bloemer, Appl. Phys. Lett. 100, 201109 (2012).

${ }^{11}$ S. M. Rytov, Theory of ElectricFluctuations and Thermal Radiation (Air Force Cambridge Research Center, Massachusetts, 1959). 
${ }^{12}$ D. Polder and M. Van Hove, Phys. Rev. B 4, 3303 (1971).

${ }^{13}$ Zh. Zhang, Nano/Microscale Heat Transfer (McGraw-Hill, Atlanta, Georgia, USA, 2007).

${ }^{14}$ J. B. Pendry, J. Phys.: Condens. Matter 11, 6621 (1999).

${ }^{15}$ I. S. Nefedov and C. R. Simovski, Phys. Rev. B 84, 195459 (2011).

${ }^{16}$ S.-A. Biehs, M. Tschikin, and P. Ben-Abdallah, Phys. Rev. Lett. 109, 104301 (2012).

${ }^{17}$ Y. Guo, C. L. Cortes, S. Molesky, and Z. Jacob, Appl. Phys. Lett. 101, 131106 (2012).

${ }^{18}$ S.-A. Biehs, M. Tschikin, R. Messina, and P. Ben-Abdallah, Appl. Phys. Lett. 102, 131106 (2013).

${ }^{19}$ B. Liu and S. Shen, Phys. Rev. B 87, 115403 (2013).

${ }^{20}$ C. Simovski, S. Maslovski, I. Nefedov, and S. Tretyakov, Opt. Express 21, 14988 (2013).

${ }^{21}$ S. Y. Lin, J. Moreno, and J. G. Fleming, Appl. Phys. Lett. 83, 380 (2003).

${ }^{22}$ T. Trupke, P. Würfel, and M. A. Green, Appl. Phys. Lett. 84, 1997 (2004).

${ }^{23}$ A. I. Volokitin and B. N. J. Persson, Rev. Mod. Phys. 79, 1291 (2007).

${ }^{24}$ G. Bimonte and E. Santamato, Phys. Rev. A 76, 013810 (2007).

${ }^{25}$ S.-A. Biehs, P. Ben-Abdallah, F. S. S. Rosa, K. Joulain, and J.-J. Greffet, Opt. Express 19, A1088 (2011).

${ }^{26}$ R. Messina and M. Antezza, Phys. Rev. A 84, 042102 (2011).

${ }^{27}$ M. Krüger, G. Bimonte, T. Emig, and M. Kardar, Phys. Rev. B 86, 115423 (2012).

${ }^{28}$ C. M. Rytov, Uspekhy Fizicheskih Nauk 63, 657 (1957).
${ }^{29}$ V. A. Golyk, M. Krüger, and M. Kardar, Phys. Rev. B 85, 046603 (2012).

${ }^{30}$ Z. Yu, N. P. Sergeant, T. Skauli, G. Zhang, H. Wang, and S. Fan, Nat. Commun. 4, 1730 (2013).

${ }^{31}$ N. M. Kroll, "Quantum theory of radiation," in Quantum Optics and Electronics, edited by C. de Witt, A. Blandin, and C. Cohen-Tannoudji (Gordon and Breach, New York, 1965).

${ }^{32}$ L. B. Felsen and N. Marcuvitz, Radiation and Scattering of Waves (Prentice-Hall, Englewood Cliffs, New Jersey, 1973).

${ }^{33}$ S. M. Hashemi and I. S. Nefedov, Phys. Rev. B 86, 195411 (2012).

${ }^{34}$ C. A. Valagiannopoulos and I. S. Nefedov, Photonics Nanostruct. Fundam. Appl. 11, 182 (2013).

${ }^{35}$ I. S. Nefedov, C. A. Valagiannopoulos, S. M. Hashemi, and E. I. Nefedov, Sci. Rep. 3, 2662 (2013).

${ }^{36}$ I. S. Nefedov, C. A. Valagiannopoulos, and L. Melnikov, J. Opt. 15, 114003 (2013).

${ }^{37}$ A. I. Volokitin and B. N. J. Persson, Phys. Rev. B 63, 205404 (2001).

${ }^{38}$ S. Basu, Z. M. Zhang, and C. J. Fu, Int. J. Energy Res. 33, 1203 (2009).

${ }^{39}$ E. M. Lifshitz and L. P. Pitaevskii, Statistical Physics: Theory of the Condensed State (Pergamon, Oxford, 1980).

${ }^{40}$ G. W. Hanson, J. Appl. Phys. 103, 064302 (2008).

${ }^{41}$ I. V. Iorsh, I. S. Mukhin, I. V. Shadrivov, P. A. Belov, and Y. S. Kivshar, Phys. Rev. B 87, 075416 (2013).

${ }^{42}$ C. F. Bohren, Am. J. Phys. 51, 323 (1983).

${ }^{43}$ H. Paul and R. Fischer, Sov. Phys. Usp. 26, 923 (1983). 